\title{
Strontium-92 half-life measurement through accurate $\gamma$-ray spectrometry
}

\author{
P. Leconte ${ }^{1, \mathrm{a}}$, J.-P. Hudelot ${ }^{2}$ and M. Antony ${ }^{2}$ \\ 1 Commissariat à l'Énergie Atomique, DEN/DER/SPRC/LEPh, 13108 Saint-Paul-lez-Durance, France \\ 2 Commissariat à l'Énergie Atomique, DEN/DER/SPEx/LPE, 13108 Saint-Paul-lez-Durance, France
}

\begin{abstract}
Many aspects of the nuclear fuel cycle require an accurate knowledge of the energy release from the decay of radioactive nuclides produced in a reactor, especially half-life data for short-lived radionuclides. Short-lived fission products are also important for fission rate distribution measurements performed in low power facilities, such as EOLE and MINERVE at CEA Cadarache, and their nuclear decay data need to be known with a high precision. For these reasons, the half-life of ${ }^{92} \mathrm{Sr}$ has been measured to solve a recently observed inconsistency with the quoted value in the nuclear data libraries: $T_{1 / 2}=2.71 \pm 0.01 \mathrm{~h}$. In this work, a new value is proposed: $T_{1 / 2}=2.594 \pm 0.005 \mathrm{~h}$. A better accuracy is achieved compared to previous evaluations. It also shows a good agreement with the most recent studies: $T_{1 / 2}=2.627 \pm 0.009 \mathrm{~h}$.
\end{abstract}

\section{Introduction}

The residual power of an irradiated reactor core after shutdown represents a limiting factor because of safety requirements imposing the need to preserve core integrity at all times. This power, along with the emitted radiation spectra, must be evaluated for a reactor operating under normal and hypothetical accident conditions. Codes have been developed to calculate the evolution of radionuclides as a function of the fuel burn-up and require accurate nuclear data, especially half-lives, fission yields and energy releases.

Moreover, major interests in advanced power concepts require the ability to predict the power distribution with high precision for fuel cycle management and increased fuel loading. For this purpose, dedicated experiments are performed in the EOLE and MINERVE facilities [1], with a specific $\gamma$-ray spectrometry technique to compare the activity of different types of fuel. This technique involves the study of fission products with an appropriate half-life for post-irradiation measurements (a few hours to a few days).

${ }^{92} \mathrm{Sr}$ is a suitable nuclide for these experiments with an intense $\gamma$-ray at $1383.9 \mathrm{keV}\left(I_{\gamma}=(93.0 \pm 0.6) \%\right)$, a high fission yield $\left(Y_{t h}=(6.034 \pm 0.066) \%\right.$ for $\left.{ }^{235} \mathrm{U}\right)$ and an adapted halflife $\left(T_{1 / 2}=2.71 \pm 0.01 \mathrm{~h}\right)$ [2].

The aim of this work is to identify an inconsistency on the reported half-life in the main nuclear data libraries, and to propose a new value with a better accuracy by the mean of several $\gamma$-ray spectrometry measurements.

\section{Previous works}

The reported half-life of ${ }^{92} \mathrm{Sr}$ in the JEF-2.2 [3], JEFF-3.1 [4], ENDF/B-VI.8 [5] and JENDL3.3 [6] nuclear data libraries is based on the measurements of Parsa [7] in 1971, reporting $T_{1 / 2}=2.71 \pm 0.01 \mathrm{~h}$. Their experimental technique consisted in the irradiation of an uranyl nitrate sample, followed by radiochemical separation of the strontium isotopes. The decay

\footnotetext{
${ }^{a}$ Presenting author, e-mail: pierre.leconte@cea.fr
}

Table 1. Previous measurements of the half-life of ${ }^{92} \mathrm{Sr}$.

\begin{tabular}{|l|l|l|l|}
\hline Reference & Year & Detector & $T_{1 / 2}(\mathrm{~h})$ \\
\hline G. Hermann [9] & 1956 & $\mathrm{NaI}$ & $2.57 \pm 0.07$ \\
\hline K. Fritze [10] & 1960 & $\mathrm{NaI}$ & $2.71 \pm 0.02$ \\
\hline B. Parsa [7] & 1971 & $\mathrm{Ge}(\mathrm{Li})$ & $2.71 \pm 0.01$ \\
\hline Y. Nir-El [8] & 2003 & $\mathrm{Ge}(\mathrm{Li})$ & $2.627 \pm 0.009$ \\
\hline
\end{tabular}

of ${ }^{92} \mathrm{Sr}$ was studied with a $\mathrm{Ge}(\mathrm{Li})$ detector and a single channel analyzer for precise gate selection of the 1383.9$\mathrm{keV}$ photopeak. Earlier data were also reported using the same technique, but they were obtained from $\mathrm{NaI}$ detectors which involved less accurate background subtraction. Thus, the inclusion of the $1405-\mathrm{keV} \gamma$-ray of ${ }^{92} \mathrm{Y}$ and the $1414 \mathrm{keV}$ $\gamma$-ray of ${ }^{91} \mathrm{Sr}$ interfered with the $\gamma$-ray of primary interest.

A new measurement was performed by Nir-El [8] in 2003 with a $\mathrm{Ge}(\mathrm{Li})$ detector and reported $T_{1 / 2}=2.627 \pm 0.009 \mathrm{~h}$. This value has been updated in the ENSDF in august 2003 [2] which now reports the weighted average of the Parsa and the Nir-El measurements: $T_{1 / 2}=2.66 \pm 0.04 \mathrm{~h}$. This value is adopted in ENDF/B-VII.0 [11]. Nevertheless, the evaluator states that this half-life recommendation may not be reliable since these data are discrepant.

\section{Analysis of the JEFF-3.1 reported value}

The inconsistency in the JEFF-3.1 value has been identified from the $\gamma$-ray spectrometry analysis [12] of $\mathrm{UO}_{2}$ fuel samples, irradiated in the MINERVE reactor, throughout two independent methods.

The first one consists in the checking of the reproducibility in the activity measurements. This activity is proportional to the saturated count rate $n_{\text {sat }}(E)$, defined as the measured count rate of the $\gamma$-ray with the energy $E$, corrected from the dead time and from the radioactive decay:

$$
n_{s a t}(E)=\frac{N(E)}{t_{m}} C_{\theta} C_{d e c}
$$


Table 2. Reproducibility in the fission rate measurement (normalized to the first value).

\begin{tabular}{|c|l|l|l|}
\hline Run number & Strontium 92 & Iodine 135 & Yttrium 91m \\
\hline 1 & $1.000 \pm 0.006$ & $1.000 \pm 0.006$ & $1.000 \pm 0.005$ \\
\hline 2 & $0.991 \pm 0.008$ & $0.984 \pm 0.007$ & $0.996 \pm 0.005$ \\
\hline 3 & $0.985 \pm 0.010$ & $1.010 \pm 0.007$ & $1.010 \pm 0.005$ \\
\hline 4 & $0.984 \pm 0.012$ & $0.997 \pm 0.008$ & $0.992 \pm 0.005$ \\
\hline 5 & $0.976 \pm 0.015$ & $0.992 \pm 0.009$ & $0.999 \pm 0.005$ \\
\hline 6 & $0.970 \pm 0.018$ & $0.999 \pm 0.010$ & $0.994 \pm 0.005$ \\
\hline
\end{tabular}

$N(E)$ is the net area of the photopeak, $t_{m}$ is the real time of measurement, $C_{\theta}$ and $C_{d e c}$ are respectively the dead time and the radioactive decay correction. In the case of a purely exponential decay, $C_{d e c}$ can be expressed as a function of the decay constant $\lambda$, the irradiation time $t_{i}$ and cooling time $t_{o}$ :

$$
C_{d e c}=\frac{\lambda t_{m}}{e^{-\lambda t_{o}}\left(1-e^{-\lambda t_{m}}\right)\left(1-e^{-\lambda t_{i}}\right)} .
$$

The following table shows a series of six successive measurements on an irradiated fuel sample, obtained for three different fission products, with an individual acquiring time of 30 minutes:

As the measurement is performed a few hours after the irradiation, no other significant $\gamma$-ray perturbates the $1383.9 \mathrm{keV}$ photopeak over an energy range of at least 3 FWHM (Full Width at Half Maximum). Therefore, one can conclude that the observed trend in these activity measurements may be linked to an overestimation of the adopted halflife.

The second method consists in comparing the fission rate measurements obtained from several fission products by means of the usual relation [13]:

$$
F=\frac{n_{\text {sat }}(E)}{Y I(E) R^{P}(E) T(E)},
$$

where $F$ is the fission rate, $Y$ the average fission yield, $I(E)$ the emission probability of the studied $\gamma$-ray, $R^{P}(E)$ the calibrated efficiency of the detector and $T(E)$ the efficiency transfer for self-absorption correction.

Irradiated fuel samples are usually measured several ${ }^{92} \mathrm{Sr}$ half-life periods after irradiation to let the activity of very short-lived $\left(T_{1 / 2}<1 \mathrm{~h}\right)$ fission products become negligible. As a consequence, the uncertainty of the fission rate is very sensitive to the uncertainty of the decay constant:

$$
\frac{\partial F}{\partial \lambda}=\left(1+\lambda t_{\mathrm{o}}-\frac{\lambda t_{i}}{e^{\lambda t_{i}}-1}-\frac{\lambda t_{m}}{e^{\lambda t_{m}}-1}\right) \frac{F}{\lambda} .
$$

As shown in the following table, the fission rate measured from ${ }^{92} \mathrm{Sr}, 18$ hours after the irradiation, is $20 \%$ lower compared with the other fission products:

This trend is consistent with the results obtained from the first method and indicates an overestimation of the halflife of ${ }^{92} \mathrm{Sr}$ beyond its reported uncertainty. An estimation of the discrepancy between the current evaluation and the "true value" can be easily calculated by means of the equation (4), considering the weighted average of the fission rate
Table 3. Comparison of fission rate measurements (arbitrary units).

\begin{tabular}{|l|l|l|}
\hline Fission product & Sample A & Sample B \\
\hline Yttrium 91m & $6.77 \pm 0.34$ & $5.93 \pm 0.30$ \\
\hline Iodine 132 & $6.71 \pm 0.21$ & $5.87 \pm 0.19$ \\
\hline Iodine 135 & $6.77 \pm 0.29$ & $5.97 \pm 0.26$ \\
\hline Cerium 143 & $6.93 \pm 0.12$ & $6.01 \pm 0.10$ \\
\hline Strontium 92 & $5.29 \pm 0.12$ & $4.71 \pm 0.09$ \\
\hline
\end{tabular}

measurements from the other fission products and solving the equation numerically. This basic procedure leads to a possible overestimation of the half-life of ${ }^{92} \mathrm{Sr}$ of about $5 \%$.

Following this preliminary study, specific measurements have been undertaken to improve the recommended half-life through minimization of the associated uncertainty.

\section{Method of measurement}

The experimental technique consists of the irradiation of two uranium-235 foils over 3 hours in a $1.5 \times 10^{8} \mathrm{n} . \mathrm{cm}^{-2} \cdot \mathrm{s}^{-1}$ flux inside a thermalized channel of the MINERVE facility. After a cooling phase of 3 hours to allow the activity of very short-lived fission products to become negligible, the samples are unloaded and counted in front of one $310 \mathrm{~cm}^{3}$ coaxial HPGe detector and one $40 \mathrm{~cm}^{3}$ planar LEGe detector. Each spectrometer consists of a resistive pre-amplifier, a DSP-2060 (amplifier + coder) and a PCA3-8K multi-channel analyzer connected to a $\mathrm{PC}$, providing data storage and analysis via the GENIE 2000 software.

Four series of 12 measurements with a constant data accumulation time of 2 hours, over a period of 9 to 13 half-lives, are carried out. Samples are axially centered with respect to the detector at a distance of 50 to $100 \mathrm{~mm}$, depending on the detector in order to have an equivalent total count rate. Dead time throughout the complete measurement phase decreases from $20 \%$ to $3 \%$.

The decay constant is estimated from a linear least-square fit of the logarithm of the measured count rates with decay time, and using statistical weights linked to the uncertainty to measure the net areas of the photopeaks. Achieving high accuracy is dependent on two main factors: the analyses of the $\gamma$-spectra (including the model adopted for the peak shape, and background subtraction), and the correctness of pulse pile-up rejection.

Three methods are employed to estimate the total area of the $1383.9 \mathrm{keV}$ photopeak of ${ }^{92} \mathrm{Sr}$ : (i) the standard summing algorithm which involves calculating the integral count in a $4.0 \times$ FWHM wide Region Of Interest (ROI) around the peak minus the integral of the background from a linear model evaluated over $1.0 \times$ FWHM at a distance of $2.0 \times$ FWHM from the centre of the peak; (ii) the fitting algorithm determines the shape of the gamma line and the background by means of a least-squares method with a $4.0 \times$ FWHM wide ROI, in which the photopeak is described by a Gaussian with an exponentially decaying tail on the left-hand side linked to the incomplete charge collection phenomena inside the germanium crystal; (iii) the same algorithm as (ii), but instead of considering the $1383.9 \mathrm{keV}$ photopeak of ${ }^{92} \mathrm{Sr}$ as a singlet, 
the nearest photopeaks from other fission products is included inside an n-multiplet from 1360 to $1400 \mathrm{keV}$.

A fixed source of ${ }^{60} \mathrm{Co}$ is placed in front of the detector at a distance of $300 \mathrm{~mm}$ to check the accuracy of the dead time correction throughout the measurements. The position of this source is adapted so that the activity contributed less than $3 \%$ to the total dead time. An additional PCA3-8K card, working in the MultiChannel Scaling mode (MCS), was also connected to the Incoming Count Rate (ICR) output of the DSP to simultaneously record the decay of the total activity of the irradiated sample and to perform a post-correction on the dead time due to the measurement of a short-lived nuclide. This step is necessary before analyzing the decay of the ${ }^{92} \mathrm{Sr}$ activity, and therefore a detailed study of the dead time corrections in the presence of varying activities is presented below.

\section{Dead time correction for short-lived nuclides}

The dead time correction used to compensate for the pulse pile-up phenomena on the DSP2060 is the so called "live time correction" which can be expressed as follows:

$$
C_{\theta}=\frac{1}{1-\theta}=\frac{t_{r}}{t_{a}},
$$

where $\theta$ is the mean dead time during the measurement, $t_{r}$ and $t_{a}$ are respectively the real and live measurement time, estimated from the amplifier stage of the electronic chain. This equation is correct under the assumption that the dead time during the measurement is nearly constant. As this is not the case for short-lived fission products, post-correction is required to compensate for the decay [14].

The first step consists in fitting a basic model to the decay curve of the total activity, recorded from the ICR output, which is done using a sum of two exponential functions with three multiplicative constants $\theta_{0}, a_{1}, a_{2}$ and two characteristic decay constants $b_{1}, b_{2}$.

As the dead time is nearly proportional to the total activity, its time dependence during the measurement of the irradiated foils can be expressed as follows :

$$
\theta(t)=\theta_{o}\left(1+\sum_{i=1}^{2} a_{i}\left(1-e^{-b_{i} t}\right)\right)
$$

The dead time estimated by the DSP 2060 during the measurement is a mean value and can be estimated from the previous relationship by using the equation:

$\bar{\theta}=\theta_{\mathrm{o}}\left(1+a_{1}\left(1-\frac{1-e^{-b_{1} t_{r}}}{b_{1} t_{r}} e^{-b_{1} t_{o}}\right)+a_{2}\left(1-\frac{1-e^{-b_{2} t_{r}}}{b_{2} t_{r}} e^{-b_{2} t_{o}}\right)\right)$.

The second step consists in solving the equation giving the measured count rate $m(t)$ as a function of the real count rate $n(t)$ without pulse pile-up $\left(n(t)=n_{\mathrm{o}} \exp (-\lambda t)\right)$ :

$$
m(t)=n(t)(1-\theta(t))
$$

Integration between $t_{\mathrm{o}}$ and $t_{\mathrm{o}}+t_{r}$, and adoption of the previous model for dead time decay as a function of time gives the $C_{\theta}$ correction expressed as follows:

$$
C_{\theta}=\frac{1}{1-\theta \xi}
$$

with the post-correction factor $\xi$ given by:

$$
\xi=\frac{1+a_{1}\left(1-\alpha_{1}\right)+a_{2}\left(1-\alpha_{2}\right)}{1+a_{1}\left(1-\frac{1-e^{-b_{1} t_{r}}}{b_{1} t_{r}} e^{-b_{1} t_{o}}\right)+a_{2}\left(1-\frac{1-e^{-b_{2} t_{r}}}{b_{2} t_{r}} e^{-b_{2} t_{o}}\right)}
$$

and $\alpha_{i}$ given by $\alpha_{i}^{=} \frac{\frac{\lambda}{\lambda+b_{i}}\left(1-e_{\left.-\left(\lambda+b_{i}\right) t_{m}\right) e_{-} b_{i} t_{0}}\right.}{1-e_{-\lambda t_{m}}}$.

In the case of a long-lived nuclide, a short measurement time, or after a sufficiently long cooling time of the irradiated sample to make the activity less time dependent, this expression tends towards $\xi=1$ which is consistent with the case of constant activity.

The activity of ${ }^{60} \mathrm{Co}$ is checked using its two main $\gamma$-ray emissions with energies $1173 \mathrm{keV}$ and $1332 \mathrm{keV}$ to estimate the accuracy of the pulse pile-up rejection circuit of the DSP. The consistency between external and internal variance indicates the correctness of the pulse pile-up rejection circuit until a $20 \%$ dead time, with an accuracy better than $0.5 \%$.

The decay of the ${ }^{92} \mathrm{Sr}$ activity is measured from the four dedicated experiments performed on fissile foils and also from the four previously irradiated fuel pins to reduce the final uncertainty. The results obtained from the fitting of each series are given in the tables below:

As the fuel pins have been measured over a time period

Table 4. Experimental results in the measurement of the ${ }^{92} \mathrm{Sr}$ decay constant (foil samples).

\begin{tabular}{|l|c|c|c|}
\hline Sample & $\begin{array}{c}\text { Analysis } \\
\text { method }\end{array}$ & $\begin{array}{c}\lambda_{i} \\
\left(\times 10^{-5} \mathrm{~s}^{-1}\right)\end{array}$ & $\begin{array}{c}\lambda_{\text {average }} \\
\left(\times 10^{-5} \mathrm{~s}^{-1}\right)\end{array}$ \\
\hline Foil 1 & (i) & $7.447 \pm 0.020$ & \\
on HPGe & (ii) & $7.423 \pm 0.025$ & $7.439 \pm 0.021$ \\
& (iii) & $7.440 \pm 0.019$ & \\
\hline Foil 2 & (i) & $7.410 \pm 0.020$ & \\
on HPGe & (ii) & $7.403 \pm 0.010$ & $7.407 \pm 0.021$ \\
& (iii) & $7.445 \pm 0.032$ & \\
\hline Foil 3 & (i) & $7.454 \pm 0.028$ & \\
on LEGe & (ii) & $7.417 \pm 0.038$ & $7.443 \pm 0.039$ \\
& (iii) & $7.452 \pm 0.052$ & \\
\hline Foil 4 & (i) & $7.414 \pm 0.032$ & \\
on LEGe & (ii) & $7.398 \pm 0.022$ & $7.402 \pm 0.026$ \\
& (iii) & $7.400 \pm 0.024$ & \\
\hline
\end{tabular}

of less than five half-lives, the measured decay constants were determined with a higher uncertainty than the measurements with the foils, and show more sensitivity to the analysis method for the net area evaluation of the photopeaks. A weighted average from the three values was determined for each fuel pin. Nevertheless, as the discrepancies were sometimes beyond their reported uncertainties, the associated uncertainty is calculated from the standard deviation between the three values, with a multiplication factor $t_{p}(v)=1.32$ [15] that arose from the low degree of freedom of the sample. 
Table 5. Experimental results in the measurement of the ${ }^{92} \mathrm{Sr}$ decay constant (fuel pin samples).

\begin{tabular}{|l|c|c|c|}
\hline Sample & $\begin{array}{c}\text { Analysis } \\
\text { method }\end{array}$ & $\begin{array}{c}\lambda_{\mathrm{i}} \\
\left(\times 10^{-5} \mathrm{~s}^{-1}\right)\end{array}$ & $\begin{array}{c}\lambda_{\text {average }} \\
\left(\times 10^{-5} \mathrm{~s}^{-1}\right)\end{array}$ \\
\hline $\begin{array}{l}\text { Fuel pin 1 } \\
\text { on LEGe }\end{array}$ & (i) & $7.408 \pm 0.060$ & \\
& (ii) & $7.571 \pm 0.152$ & $7.421 \pm 0.049$ \\
\hline $\begin{array}{l}\text { Fuel pin 2 } \\
\text { on LEGe }\end{array}$ & (i) & $7.413 \pm 0.054$ & \\
& (ii) & $7.319 \pm 0.123$ & \\
(iii) & $7.706 \pm 0.079$ & $7.561 \pm 0.231$ \\
Fuel pin 3 & (i) & $7.188 \pm 0.079$ & \\
on LEGe & (ii) & $7.254 \pm 0.068$ & $7.315 \pm 0.082$ \\
& (iii) & $7.361 \pm 0.037$ & \\
\hline Fuel pin 4 & (i) & $7.435 \pm 0.058$ & \\
on LEGe & (ii) & $7.411 \pm 0.034$ & $7.479 \pm 0.047$ \\
& (iii) & $7.502 \pm 0.018$ & \\
\hline
\end{tabular}

The results are much more consistent for the fissile foils. Weighted averages are calculated for each foil with an uncertainty estimated from the average of the standard deviations, since the three analysis methods represent three consistent ways to model the statistical sample of 12 measurements.

The uncertainty due to the pulse pile-up rejection was estimated by considering the ratio of the count rate of ${ }^{92} \mathrm{Sr}$ to the average count rate of ${ }^{60} \mathrm{Co}$ from the two main lines and checking the sensitivity on the fitted constant $\lambda$. These uncertainties contributed less than $0.15 \%$ to the uncertainty on $\lambda$.

The final estimation of the decay constant is obtained from the weighted average of the eight measurements. As these values are consistent between each other and the main contribution to the uncertainty came from the counting statistics, the standard deviation of the mean value is calculated from the pooled (or internal) variance formulae. The final mean measured half-life of ${ }^{92} \mathrm{Sr}$ is determined to be:

$$
T_{1 / 2}=2.594 \pm 0.005 \mathrm{~h} \text {. }
$$

\section{Conclusion}

A high precision measurement of the half-life of ${ }^{92} \mathrm{Sr}$ has been performed to resolve an observed discrepancy between a recent measurement and the recommended half-life to be found in evaluated nuclear applications databases. This study shows an overestimation of $4.5 \%$ of the half-life values to be found in the main nuclear applications libraries $\left(T_{1 / 2}=\right.$ $2.71 \pm 0.01 \mathrm{~h}$ ), corresponding to $12 \sigma$ beyond the associated uncertainty. The reported half-life in this work $\left(T_{1 / 2}=2.594 \pm\right.$ $0.005 \mathrm{~h}$ ) has been obtained from eight sets of measurements on irradiated fissile foils and irradiated fuel pins, and shows a consistency within $3 \sigma$ with the value reported by Nir-El. The uncertainty has been reduced by a factor of two with respect to previous studies and includes influencing factors such as pulse pile-up rejection, activity decay on the dead time correction and photopeak treatments in $\gamma$-spectrum analysis.

\section{References}

1. P. Fougeras, EOLE, MINERVE and MASURCA facilities and their associated neutron experimental programs, Proceedings of the $13^{\text {th }}$ International Conference in Nuclear Engineering, Beijing, China, May 2005.

2. C.M. Baglin, ENSDF for ${ }^{92} \mathrm{Sr}$, National Nuclear Data Center, August 2003.

3. Nuclear Energy Agency, JEF Report 17, April 2000.

4. A. Koning, The JEFF Evaluated Nuclear Data Project, Proceeding of the International Conference on Nuclear Data ND2007 (this conference).

5. Cross Section Evaluation Working Group, Berkeley National Laboratory Report NCS-44945-01/04-Rev, 2002.

6. K. Shibata, T. Kawano, T. Nakagawa, O. Iwamoto, J. Katakura, T. Fukahori, S. Chiba, A. Hasegawa, T. Murata, H. Matsunobu, T. Ohsawa, Y. Nakajima, T. Yoshida, A. Zukeran, M. Kawai, M. Baba, M. Ishikawa, T. Asami, T. Watanabe, Y. Watanabe, M. Igashira, N. Yamamuro, H. Kitazawa, N. Yamano, H. Takano, J. Nucl. Sci. Technol. 39, 1125 (2002).

7. P. Parsa, A. Ashari, L. Goolvard, YM. Nobar, Nucl. Phys. A 175, 629 (1971).

8. Y. Nir-El (private communications), Soreq research Center, Yavne, Israel.

9. G. Herrmann, Z. Naturforsch 11a, 946 (1956).

10. K. Fritze, Can. J. Phys. 38, 1614 (1960).

11. J.K. Tuli, Nucl. Data Sheets 107, $\mathrm{N}^{\circ} 12,2931$ (2006).

12. G. Gilmore, J. Hemingway, Practical Gamma-ray spectrometry (John Wiley \& Sons, 1995).

13. P. Leconte, J.-P. Hudelot, M. Antony, Integral Capture Rate Measurements of Neutron Absorbers and Actinides by $\gamma$-ray Spectrometry, Proceedings of the International Congress IYNC2006, Stockholm, Sweden, June 2006.

14. J. Adam, A.G. Belov, R. Brandt, P. Chaloun, M. Honusek, V.G. Kalinnikov, M.I. Krivopustov, B.A. Kulakov, E.J. Langrock, V.S. Pronskikh, A.N. Sosnin, V.I. Stegailov, V.M. Tsoupko-Sitnikov, J.S. Wan, W. Westmeier, Nucl. Instrum. Meth. Phys. Res. B 187, 419 (2002).

15. Bureau International des Poids et Mesures, Guide to the Expression of Uncertainty in Measurement, 1st edition, International Organization for Standardization, Geneva, Switzerland, 1995. 